\title{
The incidence of neoplasia in patients with autoimmune metaplastic atrophic gastritis: a renewed call for surveillance
}

\author{
Nadim Mahmuda, Kristen Stashek ${ }^{b}$, Bryson W. Katona ${ }^{a}$, Rashmi Tondon ${ }^{b}$, Stuti G. Shroff ${ }^{b}$, Robert Rosesc, \\ Emma E. Furth ${ }^{\text {}}$, David C. Metz ${ }^{\mathrm{a}}$
}

University of Pennsylvania, Philadelphia, PA, USA

\begin{abstract}
Background Autoimmune metaplastic atrophic gastritis (AMAG) is an immune-mediated process that may lead to pernicious anemia (PA) and an increased risk of gastric cancer. Although some literature supports 3- or 5-year endoscopic surveillance for gastric cancer in patients with PA, no formal guidance exists for the general AMAG population. We sought to identify the prevalence and incidence rates of dysplasia or adenocarcinoma in patients with AMAG in order to clarify endoscopic best practices.

Methods A retrospective study of 150 patients diagnosed with AMAG on endoscopic gastric biopsy between $1 / 2010$ and 11/2015 was performed at a tertiary medical center. Clinical and pathologic data were obtained in order to calculate the prevalence and the incidence rate of dysplasia or adenocarcinoma.

Results The cohort was predominantly female (82\%) and white (61\%), with median age 64 years. PA was present in $47 \%$ of patients. On index endoscopy, the prevalence of adenocarcinoma was $5.3 \%$. A total of 59 patients with AMAG, but without neoplasia on initial biopsy, underwent subsequent endoscopic surveillance. Two patients, both of whom had confirmed PA, developed adenocarcinoma. The incidence rate of adenocarcinoma among this group was 14.2 cases per 1000 person-years, which far exceeds that of the general population ( 0.073 per 1000 person-years) based on Surveillance, Epidemiology, and End Results data.

Conclusions AMAG is associated with a high prevalence and incidence of gastric cancer, and endoscopic surveillance should be considered. Prospective cohort studies and cost effectiveness analyses are needed to better estimate cancer risk and recommended endoscopic surveillance intervals in these patients.
\end{abstract}

Keywords Autoimmune metaplastic atrophic gastritis, incidence, gastric adenocarcinoma, surveillance, upper endoscopy

Ann Gastroenterol 2019; 32 (1): 1-6

\section{Introduction}

Autoimmune metaplastic atrophic gastritis (AMAG) is a histopathological finding that describes an immune-

aDivision of Gastroenterology (Nadim Mahmud, Bryson W. Katona, David C. Metz); ' Pathology (Kristen Stashek, Rashmi Tondon, Stuti G. Shroff, Emma E. Furth); 'Surgery (Robert Roses), University of Pennsylvania, Philadelphia, PA, United States

Conflict of Interest: Nadim Mahmud is supported by a National Institutes of Health T32 grant (2-T32-DK007740-21A1). The authors declare that they have no personal interests or other funding sources relevant to this study or manuscript

Correspondence to: Nadim Mahmud MD MS MPH, 3400 Civic Center Boulevard, $4^{\text {th }}$ Floor, South Pavilion, Philadelphia, PA 19104, USA, e-mail: nadim.mahmud@uphs.upenn.edu

Received 21 August 2018; accepted 11 October 2018; published online 08 November 2018

DOI: https://doi.org/10.20524/aog.2018.0325

(c) 2019 Hellenic Society of Gastroenterology mediated chronic gastritis. Inflammation is concentrated in the oxyntic mucosa of the corpus and fundus, resulting in destruction of parietal cells with progressive achlorhydria and hypergastrinemia [1]. Over the course of many years, and possibly decades, AMAG may thus lead to pernicious anemia (PA) [2], characterized by impaired vitamin B12 absorption and resultant macrocytic anemia. Previous studies have shown that AMAG is far more common in women than men [3] and is frequently associated with autoimmune thyroid conditions $[4,5]$.

Importantly, as a consequence of chronic inflammation, patients with AMAG have a predilection for neoplastic change. In particular, AMAG confers an increased risk of intestinal-type gastric cancer [6]. This has been described most extensively in patients who have progressed to PA, who may be at particularly high risk. In a 20 -year cohort study of over 4500 patients with discharge diagnoses of PA, a 3-fold increased risk of gastric cancer was observed [7]. However, the true annual incidence of gastric cancer in PA is controversial, with estimates ranging from $0-1.2 \%$ or more $[8,9]$. Part of this discrepancy may be related to the variable presence of Helicobacter pylori (H. pylori) 
in some of these studies, which represents an independent risk factor for gastric cancer [10]. The sum of the literature has resulted in uncertainty with respect to upper endoscopic surveillance intervals for patients with PA. Some groups have recommended surveillance at 3-5-year intervals for selected patients $[11,12]$, while other authors have concluded that there is no increased risk of gastric cancer relative to that expected in the general population and surveillance is unnecessary [13].

Moreover, the incidence of gastric adenocarcinoma with an isolated finding of AMAG on pathology (independent of PA status) is even less well-established. One study group has addressed this question, finding an incidence rate of 1.4 cases of gastric adenocarcinoma per 1000 person-years [14], and concluding that surveillance endoscopy need not be performed prior to 4 years after index biopsy [15]. Both studies were somewhat limited by small sample sizes of 106 and 44, respectively. Therefore, in the present study we sought to expand the literature by reporting our center experience. In particular, we aimed to determine the prevalence and incidence rate of gastric adenocarcinoma in patients with AMAG on pathology without concomitant $H$. pylori and independent of PA status.

\section{Patients and methods}

\section{Patient selection}

A retrospective observational study was performed at the Hospital of the University of Pennsylvania between 1/2010 and $11 / 2015$. To define the pool of patients for study, a CERNER pathology database search was conducted to identify patients with findings of AMAG on gastric biopsies obtained during upper endoscopy. At our institution, it is standard to biopsy according to the updated Sydney system for gastritis (two biopsies from the greater and lesser curvature of the antrum, two from the greater and lesser curvature of the corpus, and one from the incisura) [16], with additional biopsies performed at the discretion of the endoscopist. AMAG was defined histologically, consistently with previously published methods, based on the following criteria: 1) atrophy of the oxyntic mucosa, defined as a decrease in the parietal chief cell compartment; 2) no more than reactive gastropathy in the antrum; and 3) histologic absence of $H$. pylori. Intestinal metaplasia, where present, could not be the sole feature of atrophy $[1,17]$. Importantly, we excluded patients with concurrent histological evidence of $H$. pylori, as by definition the chronic gastritis induced by this infection is not autoimmune and our aim was to isolate the impact of AMAG alone on gastric neoplasia. We also excluded patients with tumors in proximity to the cardia if an endoscopic or pathologic diagnosis of Barrett's esophagus was noted.

\section{Chart review and data collection}

Institutional review board approval was obtained for this study protocol to pursue chart reviews for each patient. This included the collection of sociodemographic data (age, sex, race, ethnicity, smoking status: never, former, current), clinical data (PA, other concurrent autoimmune diagnoses, serum gastrin, gastric $\mathrm{pH}$ ) and endoscopic follow-up data (including dates and pathology results). PA was diagnosed based on clinician judgment, typically involving combinations of macrocytic anemia, vitamin B12 deficiency, and the presence of anti-parietal cell antibodies or anti-intrinsic factor antibodies. Pathology reports were reviewed to identify the presence of dysplasia and/or gastric adenocarcinoma.

\section{Primary and secondary analyses}

The primary outcomes of the study were the prevalence of dysplasia or adenocarcinoma on initial biopsy, and the subsequent incidence rate of dysplasia or adenocarcinoma among patients with isolated AMAG on initial biopsy. Prevalence was calculated as the number of patients with dysplasia (moderate or high-grade) or adenocarcinoma, respectively, on index biopsy divided by the total number of patients with AMAG. The incidence rate of dysplasia or adenocarcinoma was derived using only data from patients with longitudinal endoscopic follow up. This was computed as the number of incident dysplasia or adenocarcinoma diagnoses (based on gastric biopsy) divided by the total duration of endoscopic follow up in the longitudinal cohort (in years). Analyses were also stratified according to the presence or absence of a preexisting diagnosis of PA. Secondary analysis included the comparison of sociodemographic and clinical variables among patients with dysplasia or adenocarcinoma, in addition to multivariable logistic regression to identify significant predictors.

\section{Statistical analysis}

Normality in the data was not assumed given the relatively small sample size. Therefore, medians and interquartile ranges (IQRs) were computed, and Wilcoxon rank-sum or Fisher's exact tests were performed for statistical inference, where applicable. Multivariable logistic regression was performed to identify risk factors for prevalent dysplasia or adenocarcinoma, using forward and backwards stepwise selection for final model fitting. An a level of 0.05 was used as the threshold for statistical significance in all cases. Where relevant, missing elements of data were assumed to be missing completely at random, and were excluded from analysis in list-wise fashion. All data management and computations were performed with STATA/IC version 14.2.

\section{Results}

\section{Cohort definition and patient characteristics}

The CERNER pathology database search returned a total of 155 patients with biopsy-confirmed AMAG. Of these patients, 5 were excluded from further analysis as detailed in Fig. 1, 
leaving 150 patients for analysis. The baseline characteristics of the study cohort are summarized in Table 1 . The patients were predominantly female $(80.7 \%)$ and white $(61.3 \%)$, with a median age of 63.5 years (IQR 52.0, 72.0). A diagnosed autoimmune disease was present in $53.3 \%$ of patients, with thyroiditis being the most common (46.7\%). PA was present in $46.7 \%$ of the cohort.

\section{Primary analysis}

Among the 150 patients identified with AMAG on index gastric pathology, the prevalence of adenocarcinoma was $5.3 \%$ (95\% confidence interval [CI] 2.3-10.2\%), and the prevalence of moderate or high-grade dysplasia was $1.3 \%$ (95\%CI 0.2 $4.7 \%$ ). A preexisting diagnosis of PA was present in $50 \%$ and $100 \%$ of these patients, respectively. Of the patients with AMAG on index biopsy but no evidence of neoplasia, 59 underwent longitudinal endoscopic surveillance. The median follow-up duration was 1.89 years, with a total follow-up duration of 140.5 years. During this time, two patients (both of whom had PA) developed interval gastric adenocarcinoma, yielding an incidence rate of 14.2 cases per 1000 persons per year (95\%CI 1.7-51.4 cases per 1000 person-years). The interval diagnosis of adenocarcinoma occurred at 76.7 months (6.4 years) after index gastric biopsy in one patient, and at 75.2 months ( 6.3 years) in the other. There were no diagnoses of incident dysplasia.

\section{Secondary analysis}

Among the entire cohort, 12 patients had either dysplasia or adenocarcinoma during the observation period. Patients with dysplasia or adenocarcinoma were found to be

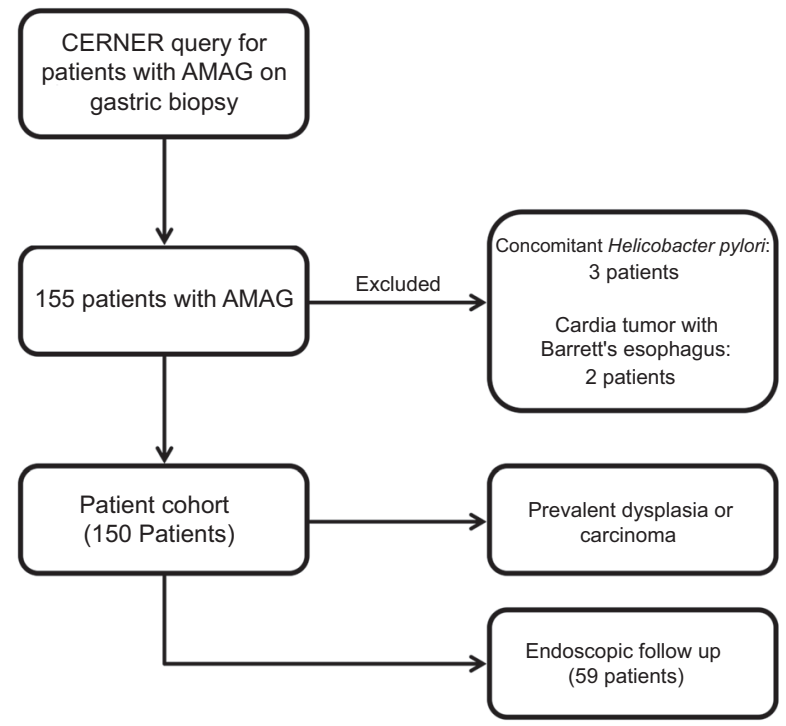

Figure 1 Patient flow diagram AMAG, autoimmune metaplastic atrophic gastritis significantly older as compared to those without (75.5 vs. 62.0 years, $\mathrm{P}=0.005)$. Age $\geq 70$ years was associated with a 5.65 -fold increased risk of dysplasia or adenocarcinoma compared to patients aged $<70$ years $(95 \% \mathrm{CI}$ for relative risk $1.60-19.98, \mathrm{P}=0.0037)$. There were no significant differences among the groups in terms of sex, race, ethnicity, smoking status, minimum gastric $\mathrm{pH}$, maximum serum gastrin, $\mathrm{PA}$, or the presence of other concomitant autoimmune disease (Table 2). On multivariable logistic regression analysis to predict prevalent dysplasia or adenocarcinoma, both forward and backward stepwise modeling methods only retained age as a significant risk factor (odds ratio per 5-year increase in age: $1.41,95 \% \mathrm{CI} 1.07-1.85, \mathrm{P}=0.013)$. Adjusted probabilities of prevalent dysplasia or adenocarcinoma are presented in Fig. 2. Sex, race, smoking status, PA, and any other autoimmune condition were not statistically significant risk factors.

Table 1 Baseline characteristics of patients with autoimmune metaplastic atrophic gastritis

\begin{tabular}{lc}
\hline Variable & Value (N=150) \\
\hline Age, median (interquartile range) & $63.5(52.0,72.0)$ \\
Sex & \\
Female & $121(80.7 \%)$ \\
Male & $29(19.3 \%)$ \\
Race & \\
White & $92(61.3 \%)$ \\
Black & $39(26.0 \%)$ \\
Asian & $8(5.3 \%)$ \\
Other & $11(7.3 \%)$ \\
Ethnicity & \\
Non-Hispanic & $145(96.7 \%)$ \\
Hispanic & $5(3.3 \%)$ \\
Smoking & \\
Never & $86(59.3 \%)$ \\
Former & $52(35.9 \%)$ \\
Current & $7(4.8 \%)$ \\
Pernicious anemia (\%) & $70(46.7 \%)$ \\
Concomitant autoimmune diagnosis & \\
Hypothyroidism (\%) & $70(46.7 \%)$ \\
Rheumatoid arthritis (\%) & $2(1.3 \%)$ \\
Hyperparathyroidism (\%) & $2(1.3 \%)$ \\
Multiple sclerosis (\%) & $2(1.3 \%)$ \\
Other (\%) & $2(1.3 \%)$ \\
\hline & $2(1.3 \%)$ \\
\hline & $8.3 \%)$ \\
\hline
\end{tabular}


Table 2 Patient characteristics by dysplasia/adenocarcinoma status

\begin{tabular}{|c|c|c|c|}
\hline \multirow[t]{2}{*}{ Factor } & \multicolumn{2}{|c|}{ Dysplasia or adenocarcinoma } & \multirow[t]{2}{*}{ P-value } \\
\hline & No $(\mathrm{N}=138)$ & Yes $(\mathrm{N}=12)$ & \\
\hline Age, median (interquartile range) & $62(52,71)$ & $75.5(66.5,81.5)$ & $0.005^{\star}$ \\
\hline Sex & & & 0.250 \\
\hline Female & $113(81.9 \%)$ & $8(66.7 \%)$ & \\
\hline Male & $25(18.1 \%)$ & $4(33.3 \%)$ & \\
\hline Race & & & 1.000 \\
\hline White & $84(60.9 \%)$ & $8(66.7 \%)$ & \\
\hline Black & $36(26.1 \%)$ & $3(25.0 \%)$ & \\
\hline Asian & $8(5.8 \%)$ & $0(0.0 \%)$ & \\
\hline Other & $10(7.2 \%)$ & $1(8.3 \%)$ & \\
\hline Ethnicity & & & 1.000 \\
\hline Non-Hispanic & $133(96.4 \%)$ & $12(100.0 \%)$ & \\
\hline Hispanic & $5(3.6 \%)$ & $0(0.0 \%)$ & \\
\hline Smoking & & & 0.620 \\
\hline Never & $80(59.7 \%)$ & $6(54.5 \%)$ & \\
\hline Former & $48(35.8 \%)$ & $4(36.4 \%)$ & \\
\hline Current & $6(4.5 \%)$ & $1(9.1 \%)$ & \\
\hline Min gastric $\mathrm{pH}$, median (IQR) & $7(6.5,7)$ & $7(7,7)$ & 0.550 \\
\hline Max serum gastrin, median (IQR) & $834(510,13915)$ & $868(868,868)$ & 0.960 \\
\hline Pernicious anemia & & & 1.000 \\
\hline No & $74(53.6 \%)$ & $6(50.0 \%)$ & \\
\hline Yes & $64(46.4 \%)$ & $6(50.0 \%)$ & \\
\hline Concomitant autoimmune disease & & & 1.000 \\
\hline No & $64(46.4 \%)$ & $6(50.0 \%)$ & \\
\hline Yes & $74(53.6 \%)$ & $6(50.0 \%)$ & \\
\hline
\end{tabular}

* Statistically significant at the $\alpha=0.05$ level

$I Q R$, interquartile range

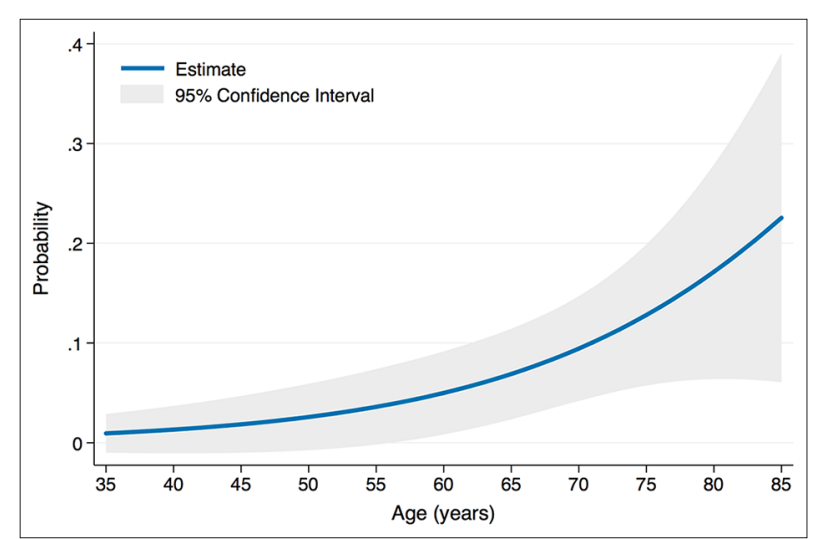

Figure 2 Probability of dysplasia or adenocarcinoma on index autoimmune metaplastic atrophic gastritis biopsy by age

\section{Discussion}

In this study of 150 patients with findings of AMAG on gastric biopsy, we found a high prevalence (5.3\%) and incidence of gastric adenocarcinoma ( 14.2 cases per 1000 person-years, or $1.4 \%$ annual incidence). The high prevalence strongly suggests that patients with potential AMAG should undergo early upper endoscopic evaluation. As AMAG itself often presents with vague dyspepsia or without symptoms [18], a high index of suspicion (i.e., based on associated autoimmune diagnoses or family history) and a low threshold for endoscopy are critical. Regarding the high incidence of adenocarcinoma in the AMAG population, the risk is many-fold higher than that expected in the general population. Based on data from the Surveillance, Epidemiology, and End Results (SEER) program, the annual incidence of gastric cancer in the United States is estimated to be 0.073 cases per 1000 person-years [19]. In fact, the estimates of gastric cancer risk associated with $H$. pylori-negative AMAG in this study are even on a par with or slightly greater than those seen with isolated $H$. pylori infection, which confers a 10 to 20 -fold relative risk based on epidemiological studies [20-22]. While our cohort is a highly-selected population, it represents a group for whom there remains uncertainty with respect to endoscopic surveillance practices and prognostication. 
Relative to other studies evaluating the risk of gastric cancer in non- $H$. pylori AMAG patients, the prevalence and incidence of gastric neoplasia in our study were somewhat higher. A large study by Park et al identified 11 adenocarcinomas among 461 patients with AMAG, for a crude prevalence of 2.4\% [23]. Separately, as noted previously, prior literature has estimated an annual incidence of gastric cancer of $0-1.2 \%$ for patients with PA [12], with a pooled estimate of $0.27 \%$ per person-year in a recent meta-analysis [9]. There are several possible reasons for the differences observed. First, the patients in our study were older (median age 63.5 years) than those in many prior studies $[8,24]$. Indeed, we noted that older patients ( $\geq 70$ years) were more likely to have gastric neoplasia and that increasing age portends a higher risk of dysplasia or adenocarcinoma on index biopsy. Second, we had incomplete endoscopic surveillance data in our study (detailed below), which may have caused an overestimation of the incidence rate. Third, given the small sample size in these studies, with a low event rate, estimates of incidence rates are very sensitive to incremental changes in events-this is reflected in the wide confidence interval for incidence rate. However, it is important to highlight here that much of the literature to date has focused exclusively on patients with PA. A relative strength of our study is that it identifies a cohort of patients with a histopathological diagnosis of AMAG, a necessary precursor for PA. The estimates produced in our study, therefore, represent a more heterogeneous population than has been typically studied previously.

Regarding the time to development of gastric cancer in those patients without neoplasia on initial biopsy, both patients with incident adenocarcinoma were diagnosed after 6 years of follow up. Based on these data, an endoscopic follow-up interval of three years appears reasonable, as has been suggested previously in the literature [11,25]. This window would ideally allow for the detection of precancerous lesions (i.e., dysplasia) rather than incident adenocarcinomas. Our data further suggest that patients aged 70 and older are at particularly high risk of adenocarcinoma, and increased vigilance is warranted in this setting. In addition to clinical best practice, these considerations are also of great import to the cost-effectiveness of endoscopic surveillance, which has been an active area of research in similar settings, such as gastric intestinal metaplasia [26]. Finally, it is worthwhile to note that our endoscopic recommendations for AMAG patients would be less stringent than existing guidelines for comparable disease processes. For example, the American Society for Gastrointestinal Endoscopy currently recommends endoscopic surveillance every 3-5 years for patients with non-dysplastic Barrett's esophagus who would be eligible for treatment [27]. This is despite an annual incidence of esophageal cancer of only approx. $0.3 \%$ in these patients. Based on existing precedent, therefore, a three-year endoscopic surveillance interval for AMAG patients is justifiable.

There are several limitations to note in this study. First, the incidence rate of adenocarcinoma was calculated considering only those patients who underwent endoscopic surveillance. There were 81 additional patients identified as having AMAG on initial pathology who did not undergo further endoscopic evaluation during the study period, and it is unknown whether

\section{Summary Box}

\section{What is already known:}

- Autoimmune metaplastic atrophic gastritis (AMAG) is a form of chronic gastritis that may result in pernicious anemia (PA)

- AMAG with PA is known to increase the risk of gastric neoplasia over time

- Estimates of annual gastric cancer incidence in PA patients range from $0-1.2 \%$

- The incidence of gastric cancer in Helicobacter pylori negative patients with AMAG is poorly defined

\section{What the new findings are:}

- AMAG is associated with a high prevalence of dysplasia or gastric cancer $(5.3 \%)$

- Increasing age is associated with a significantly increased risk of prevalent dysplasia or gastric cancer in patients with AMAG

- In patients with AMAG, the incidence of gastric cancer may be higher than previously thought (14.2 per 1000 person-years in this study)

or not this group of patients developed gastric adenocarcinoma. It is conceivable that those patients who underwent endoscopic surveillance were considered to be higher risk for other reasons, such as interval symptoms suggestive of new gastric pathology (i.e., anemia, weight loss, dyspepsia, etc.). This could serve to inflate the estimated incidence rate of gastric cancer in this study. Second, there is a risk of misclassification of outcomes in the cohort who received endoscopic follow up. Lesions representing focal carcinoma may have been missed. This misclassification would favor biasing the incidence rate estimate towards zero. Third, there is possible misclassification of H. pylori status. Although histology was used to exclude patients with $H$. pylori, it is possible that some included patients may have had false negative testing. This could have the effect of inflating neoplasia estimates; however, our methods are consistent with prior literature studying AMAG [17,22]. Fourth, gastritis staging systems, such as the Operative Link on Gastritis Assessment (OLGA) or Operative Link on Gastritis/ Intestinal-Metaplasia Assessment (OLGIM), were not used in this study. It is possible, for example, that different degrees of gastritis severity portend different degrees of increased risk for gastric neoplasia. This is a potential area for future inquiry. Finally, the durations of AMAG and PA were not clearly known in this study. Some patients diagnosed with AMAG may have had these findings for many years or even decades prior. Assuming that there is a cumulative risk associated with AMAG and PA, this may have impacted estimates obtained in this study, potentially in an unpredictable fashion. 
In conclusion, our analysis suggests that AMAG is associated with a high prevalence and incidence of gastric cancer, and endoscopic surveillance should be considered in all patients with AMAG, especially in patients with advanced age ( $\geq 70$ years). While endoscopic surveillance at 3 years appears reasonable, prospective cohort studies and cost effectiveness analyses are clearly needed to further define the estimated risk of cancer in patients with AMAG and to refine recommended surveillance intervals.

\section{References}

1. Park JY, Lam-Himlin D, Vemulapalli R. Review of autoimmune metaplastic atrophic gastritis. Gastrointest Endosc 2013;77:284-292.

2. Lahner E, Annibale B. Pernicious anemia: new insights from a gastroenterological point of view. World J Gastroenterol 2009;15:5121-5128.

3. Neumann WL, Coss E, Rugge M, Genta RM. Autoimmune atrophic gastritis-pathogenesis, pathology and management. Nat Rev Gastroenterol Hepatol 2013;10:529-541.

4. Lahner E, Centanni M, Agnello G, et al. Occurrence and risk factors for autoimmune thyroid disease in patients with atrophic body gastritis. Am J Med 2008;121:136-141.

5. Centanni M, Marignani M, Gargano L, et al. Atrophic body gastritis in patients with autoimmune thyroid disease: an underdiagnosed association. Arch Intern Med 1999;159:1726-1730.

6. Lahner E, Esposito G, Galli G, Annibale B. Atrophic gastritis and pre-malignant gastric lesions. Transl Gastrointest Cancer 2015;4:272-281.

7. Hsing AW, Hansson LE, McLaughlin JK, et al. Pernicious anemia and subsequent cancer. A population-based cohort study. Cancer 1993;71:745-750.

8. Kokkola A, Sjöblom SM, Haapiainen R, Sipponen P, Puolakkainen P, Järvinen $\mathrm{H}$. The risk of gastric carcinoma and carcinoid tumours in patients with pernicious anaemia. A prospective follow-up study. Scand J Gastroenterol 1998;33:88-92.

9. Vannella L, Lahner E, Osborn J, Annibale B. Systematic review: gastric cancer incidence in pernicious anaemia. Aliment Pharmacol Ther 2013;37:375-382.

10. Hsu PI, Lai KH, Hsu PN, et al. Helicobacter pylori infection and the risk of gastric malignancy. Am J Gastroenterol 2007;102:725-730.

11. Sjöblom SM, Sipponen P, Miettinen M, Karonen SL, Järvinen HJ. Gastroscopic screening for gastric carcinoids and carcinoma in pernicious anemia. Endoscopy 1988;20:52-56.

12. Lahner E, Esposito G, Pilozzi E, et al. Occurrence of gastric cancer and carcinoids in atrophic gastritis during prospective long-term follow up. Scand J Gastroenterol 2015;50:856-865.

13. Schafer LW, Larson DE, Melton LJ $3^{\text {rd }}$, Higgins JA, Zinsmeister AR.
Risk of development of gastric carcinoma in patients with pernicious anemia: a population-based study in Rochester, Minnesota. Mayo Clin Proc 1985;60:444-448.

14. Lahner E, Bordi C, Cattaruzza MS, et al. Long-term follow-up in atrophic body gastritis patients: atrophy and intestinal metaplasia are persistent lesions irrespective of Helicobacter pylori infection. Aliment Pharmacol Ther 2005;22:471-481.

15. Lahner E, Caruana P, D’Ambra G, et al. First endoscopic-histologic follow-up in patients with body-predominant atrophic gastritis: when should it be done? Gastrointest Endosc 2001;53:443-448.

16. Yantiss RK, Odze RD. Optimal approach to obtaining mucosal biopsies for assessment of inflammatory disorders of the gastrointestinal tract. Am J Gastroenterol 2009;104:774-783.

17. Zhang H, Jin Z, Cui R, Ding S, Huang Y, Zhou L. Autoimmune metaplastic atrophic gastritis in Chinese: a study of 320 patients at a large tertiary medical center. Scand J Gastroenterol 2017;52:150-156.

18. Carabotti M, Lahner E, Esposito G, Sacchi MC, Severi C, Annibale B. Upper gastrointestinal symptoms in autoimmune gastritis: A crosssectional study. Medicine (Baltimore) 2017;96:e5784.

19. National Cancer Institute. Cancer Stat Facts: Stomach Cancer. Available from: https://seer.cancer.gov/statfacts/html/stomach. html [Accessed October 22, 2018].

20. Sipponen P, Riihelä M, Hyvärinen H, Seppälä K. Chronic nonatrophic ('superficial') gastritis increases the risk of gastric carcinoma. A casecontrol study. Scand J Gastroenterol 1994;29:336-340.

21. Ekström AM, Held M, Hansson LE, Engstrand L, Nyrén O. Helicobacter pylori in gastric cancer established by CagA immunoblot as a marker of past infection. Gastroenterology 2001;121:784-791.

22. Brenner H, Arndt V, Stegmaier C, Ziegler H, Rothenbacher D. Is Helicobacter pylori infection a necessary condition for noncardia gastric cancer? Am J Epidemiol 2004;159:252-258.

23. Park JY, Cornish TC, Lam-Himlin D, Shi C, Montgomery E. Gastric lesions in patients with autoimmune metaplastic atrophic gastritis (AMAG) in a tertiary care setting. Am J Surg Pathol 2010;34:1591-1598.

24. Sjöblom SM, Sipponen P, Järvinen H. Gastroscopic follow up of pernicious anaemia patients. Gut 1993;34:28-32.

25. Dinis-Ribeiro M, Areia M, De Vries AC, et al. Management of precancerous conditions and lesions in the stomach (MAPS): guideline from the European Society of Gastrointestinal Endoscopy (ESGE), European Helicobacter Study Group (EHSG), European Society of Pathology (ESP), and the Sociedade Portuguesa de Endoscopia Digestiva (SPED). Endoscopy 2012;44:74-94.

26. Hassan C, Zullo A, Di Giulio E, et al. Cost-effectiveness of endoscopic surveillance for gastric intestinal metaplasia. Helicobacter 2010;15:221-226.

27. Evans JA, Early DS, Fukami N, et al; Standards of Practice Committee of the American Society for Gastrointestinal Endoscopy. The role of endoscopy in Barrett's esophagus and other premalignant conditions of the esophagus. Gastrointest Endosc 2012;76:1087-1094. 